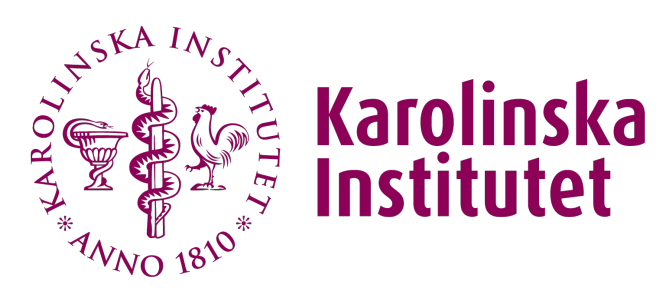

Karolinska Institutet

http://openarchive.ki.se

This is a Peer Reviewed Accepted version of the following article, accepted for publication in Cancer Epidemiology.

\title{
Time trends in the incidence of
} oesophageal cancer in Asia : \section{variations across populations and} histological types

Xie, Shao-Hua; Lagergren, Jesper

Cancer Epidemiol. 2016 Oct;44:71-76.

http://doi.org/10.1016/j.canep.2016.08.002

http://hdl.handle.net/10616/45593

If not otherwise stated by the Publisher's Terms and conditions, the manuscript is deposited under the terms of the Creative Commons Attribution-NonCommercial-NoDerivatives License (http://creativecommons.org/licenses/by-nc-nd/4.0/), which permits non-commercial re-use, distribution, and reproduction in any medium, provided the original work is properly cited, and is not altered, transformed, or built upon in any way. 
Time trends in the incidence of oesophageal cancer in Asia: variations across populations and histological types

Authors: Shao-Hua Xie ${ }^{\text {a, }}$ and Jesper Lagergren ${ }^{\text {a, b }}$

Affiliations: ${ }^{a}$ Upper Gastrointestinal Surgery, Department of Molecular Medicine and Surgery, Karolinska Institutet, Karolinska University Hospital, Stockholm, Sweden; ${ }^{\mathrm{b}}$ Section of Gastrointestinal Cancer, Division of Cancer Studies, King's College London, United Kingdom

*Corresponding authour: Upper Gastrointestinal Surgery, Department of Molecular Medicine and Surgery, Karolinska Institutet, NS 67, 2nd Floor, 17176 Stockholm, Sweden. Tel: +468517 70917; Fax: +468517 76280.

E-mail address: shaohua.xie@ki.se (S.-H. Xie). 


\section{Abstract}

Objective: We aimed to assess temporal trends in incidence rates of oesophageal cancer in Asian countries.

Materials and Methods: Using data from the Cancer Incidence in Five Continents series, we examined the temporal trends in incidence rates of oesophageal cancer by population and histological type in seven Asian countries in 1988 to 2007. Age-period-cohort analyses estimated the overall annual percentage changes (net drifts) and their $95 \%$ confidence intervals (CIs) in incidence rates.

Results: The age-standardised incidence rate of oesophageal cancer declined in most Asian populations, but remained relatively unchanged in Japan and Israel. The rate of oesophageal squamous cell carcinoma decreased in Hong Kong, Singapore and Israel, but was stable in Japan. The net drifts were statistically significant in men in Hong Kong (-3.4\%, 95\% CI: $6.1 \%,-0.7 \%)$ and in women in Singapore $(-10.1 \%, 95 \%$ CI: $-14.4 \%,-5.5 \%)$. The agestandardised incidence rates of oesophageal adenocarcinoma were below 2 and 0.5 per 100 000 in men and women, respectively, across all periods in the all registers containing valid data on histological type. The age-standardised incidence rate of oesophageal adenocarcinoma slightly increased in Japan, Singapore, and Israel, although the net drift was statistically significant only in Israeli men $(4.9 \%, 95 \%$ CI: 0.8\%, $9.1 \%)$.

Conclusion: The overall incidence rates of oesophageal cancer declined in most Asian countries, which is due to a decrease in oesophageal squamous cell carcinoma incidence. However, attention needs to be paid to a probable beginning of an increasing incidence of oesophageal adenocarcinoma in Asia.

Key words: Oesophageal cancer; Incidence; Epidemiology; Adenocarcinoma; Asia 
Abbreviations: ASR, age-standardized incidence rates; BMI, body mass index; CI,

confidence interval; OAC: oesophageal adenocarcinoma; OSCC: oesophageal squamous cell carcinoma; H. pylori, Helicobacter pylori. 


\section{Background}

Oesophageal cancer is the eighth most common type of malignancy and the sixth leading cause of cancer deaths globally [1, 2]. It was estimated that there were 456000 new cases of oesophageal cancer worldwide in 2012, among which 340000 (75\%) occurred in Asia. Men in Eastern Asia have the highest incidence rate of oesophageal cancer globally (17 per $100000)[2]$.

The risk factors for the two main histological types of oesophageal cancer, squamous cell carcinoma and adenocarcinoma, differ greatly. Oesophageal squamous cell carcinoma is mainly associated with tobacco smoking, heavy alcohol use, and certain dietary factors, while the main risk factors for oesophageal adenocarcinoma include gastro-oesophageal reflux disease , obesity, and Helicobacter pylori (H. pylori) infection (inverse association) [3-6]. Oesophageal squamous cell carcinoma is the predominant type in Asia, Africa and South America, and accounts for approximately $90 \%$ of all oesophageal cancer cases worldwide [2, 3]. In contrast, oesophageal adenocarcinoma has become the predominant type in most North American and European countries. The past four decades have witnessed a rapidly increased incidence of oesophageal adenocarcinoma in Western populations, particularly in white males [3-5].

Asian societies have experienced substantial socio-economic development with changes in the exposures in relation to cancer risk during the past several decades [7, 8]. A decreasing prevalence of tobacco smoking may have, for example, contributed to a declining risk of oesophageal squamous cell carcinoma, while an increased prevalence of obesity or reflux may have led to a rise in the incidence of oesophageal adenocarcinoma. To provide an update on potential differences in incidence of oesophageal cancer between Asian countries and changes 
in incidence over time, we analysed the incidence of oesophageal cancer in selected Asian countries by histological type using data from the Cancer Incidence in Five Continents series. 


\section{Methods}

\subsection{Data sources}

We extracted data on the incidence of oesophageal cancer and population sizes from the Cancer Incidence in Five Continents (CI5) series volumes VII to X. These are published by the International Agency for Research on Cancer (IARC) and contain information on cancer incidence worldwide where good quality data are available [9-12]. We followed the geographical definition of the United Nations, which was also in line with the practice in CI5 series [13]. We included registers in seven Asian countries with available data in all four CI5 volumes during the period 1988-2007: China (Shanghai and Hong Kong), Japan (Miyagi, Nagasaki, and Osaka), India (Chennai and Mumbai), Thailand (Chiang Mai), Singapore, Philippines (Manila), and Israel. We pooled the numbers of cases and population sizes at risk from multiple regional registers within the country for Japan and India. Evaluation of incidence by histological type was restricted to registers with a microscopically verified percentage of cases of over $80 \%$ in each of the four CI5 volumes (Supplementary Figure 1).

\subsection{Statistical analyses}

We first calculated the sex-specific crude and age-standardised incidence rates (ASRs) by population and histological type for each CI5 volume in five-year calendar periods. The ASRs were calculated using the direct method with the World Health Organisation (WHO) World Standard Population 2000 as the reference [14]. The 95\% confidence intervals (CIs) of crude rates were computed under the assumption of Poisson distribution, while CIs for ASRs were estimated based on the gamma distribution, as it assumes that the standardised rate is a weighted sum of independent Poisson random variables $[15,16]$. All these statistical analyses were performed using the statistical software SAS version 9.4 (SAS Institute, Cary, NC). 
We further performed age-period-cohort regressions on the incidence of oesophageal cancer by population and histological type for each sex using a web tool, newly developed by the National Cancer Institute (NCI), United States [17]. This web tool fits the age-period-cohort model which attempts to differentiate the effects of age, calendar period, and birth cohort on disease rates, and estimates the overall annual percentage changes (net drifts) in ASRs in addition $[17,18]$. The central age group, calendar period, and birth cohort were defined as the reference in all age-period-cohort analyses. 


\section{Results}

\subsection{Oesophageal cancer of all histological types}

In total, 53206 men and 18382 women were diagnosed with oesophageal cancer as recorded in all included registers during the period 1988-2007. The ASRs in men were generally highest in Eastern Asia, followed by India and Singapore, and were relatively low in Thailand, Philippines, and Israel (Table 1). The ASRs in women were highest in India, followed by China, and were lower (<3 per 100000 person-years) in the other populations (Table 1).

The ASRs of oesophageal cancer declined in both sexes in most Asian populations during the study period, but remained relatively stable in Japan and Israel (Figures 1). The decreases in net drifts were statistically significant in men in Shanghai $(-3.4 \%, 95 \%$ CI: $-5.3 \%,-1.4 \%)$, Hong Kong (-6.2\%, 95\% CI: $-7.8 \%,-4.6 \%)$, and India $(-1.9 \%, 95 \%$ CI: $-3.7 \%,-0.1 \%)$. The net drifts in women showed decreasing incidence rates with statistical significance in all populations, except for Japan $(2.9 \%, 95 \%$ CI: $-1.5 \%, 7.6 \%)$ and Israel $(-0.4 \%, 95 \%$ CI: $-4.1 \%$, $3.5 \%$ ) (Figure 2 and Supplementary Table 1).

\subsection{Oesophageal squamous cell carcinoma}

The incidence rates of oesophageal squamous cell carcinoma were the highest in Hong Kong and Japan, followed by Singapore, and were the lowest in Israel (Figure 3 and Supplementary Table 2).

The ASRs of oesophageal squamous cell carcinoma declined in Hong Kong, Singapore and Israel, but remained stable in Japan in both sexes (Figure 3). The net drifts were statistically significant in men in Hong Kong $(-3.4 \%, 95 \%$ CI: $-6.1 \%,-0.7 \%)$ and in women in Singapore $(-10.1 \%, 95 \%$ CI: $-14.4 \%,-5.5 \%)$ (Figure 3 and Supplementary Table 1). 


\subsection{Oesophageal adenocarcinoma}

No evident difference in the incidence rates of oesophageal adenocarcinoma between the studied Asian populations was seen except for relatively higher rates in Israel since 1993 . The ASRs of oesophageal adenocarcinoma were below 2 and 0.5 per 100000 in men and women, respectively, across calendar periods in all studied Asian populations (Figure 3 and Supplementary Table 3).

The incidence rates increased in Japan, Singapore, and Israel, although only the net drift in Israeli men was statistically significant $(4.9 \%, 95 \%$ CI: $0.8 \%, 9.1 \%)$. The percentages of oesophageal adenocarcinoma in all cases of oesophageal cancer increased from $3.6 \%$ to $11.0 \%$ and from $13.1 \%$ to $37.0 \%$ in Singapore and Israel, respectively, from the period 1988-1992 to the period 2003-2007 (Supplementary Figure 1). On the contrary, the ASRs of oesophageal adenocarcinoma significantly declined in both sexes in Hong Kong during the study period (net drifts $-8.8 \%$ and $-8.5 \%$ in men and in women, respectively).

\subsection{Unspecified and other histological types}

The rate of unspecified histology slightly declined with later calendar periods, while the rate of other histological types remained stable over time. However, the rate of unspecified histology was low and did not substantially influence the findings for squamous cell carcinoma or adenocarcinoma (Supplementary Figure 1). 


\section{Discussion}

The present study provided an update on the changing incidence of oesophageal cancer over a 20-year period in Asia, and revealed variations across populations and histological types. The incidence of oesophageal cancer declined in most Asian countries included in this analysis, but remained stable in Japan and Israel. This decline was predominantly in oesophageal squamous cell carcinoma. There was a seeming trend towards a rise in the incidence of oesophageal adenocarcinoma in Singapore and Israel.

The observed decline in the incidence of oesophageal cancer predominantly in oesophageal squamous cell carcinoma is likely to be associated with the decreasing prevalence of tobacco smoking over the past few decades in these Asian populations [19-21]. However, the stable incidence of oesophageal cancer in Japan did not parallel the decreasing prevalence of smoking in this population. It is possible that the influence of a decreasing prevalence of smoking has been neutralised by the considerable rise in alcohol consumption in the Japanese population $[21,22]$. The annual alcohol consumption per capita in people aged 15 and above in Japan increased from less than 5 litres in the 1960s to 8 litres in 2003-2005 [22]. Moreover, consumption of spirits, which is more strongly associated with the risk of oesophageal squamous cell carcinoma than other alcoholic beverages, alone accounted for over half of the total alcohol consumption in Japan [23]. Therefore, efforts to reduce the alcohol use could decrease the burden of oesophageal cancer in the Japanese population.

The incidence of oesophageal adenocarcinoma has increased rapidly in many Western populations, including North America and Europe, during the past four decades [4, 5], whereas data from Asian countries are limited. Earlier studies have shown a statistically nonsignificant rise in the incidence of oesophageal adenocarcinoma in Singapore [24], declined incidence of oesophageal adenocarcinoma and ratio of adenocarcinoma versus squamous cell 
carcinoma in Hong Kong [25], and a stable incidence in Japan and Taiwan during the past few decades [26, 27]. A recent systematic review of Barrett's oesophagus, regarded as a premalignant condition for adenocarcinoma of the oesophagus, suggested an increasing prevalence of this condition in Eastern Asian countries between 1991 and 2014 [28]. The present study with updated data till the year 2007 indicates a rise in the incidence of oesophageal adenocarcinoma and considerably increased ratio of adenocarcinoma versus squamous cell carcinoma in Singapore and Israel. This may be explained by the associated rise in the prevalence of reflux and obesity, and the decreased prevalence of $H$. pylori infection in these populations [29-32]. On the other hand, the observed increase may, at least partially, be explained by the improvements in histological typing and diagnostic procedures over time as shown in other populations $[5,33]$. Such speculation is also supported by some statistically significant period effects in males from age-period-cohort regressions (Supplementary Figure 2). However, these statistical findings need to be interpreted with caution given the known co-linearity between effects from calendar periods and birth cohorts $[17,18]$. Possible misclassification between adenocarcinomas of the oesophagus and gastric cardia might have affected the reported incidence rates, but does not seem to influence an overall interpretation of the time trends for either of these cancer sites [34]. The decreasing trend in the incidence of oesophageal adenocarcinoma in Hong Kong differs from observations in Western and other Asian populations, and is also unexpected in a population with an increasing prevalence of gastro-oesophageal reflux disease and obesity $[29,35]$, and a decreasing prevalence of $H$. pylori infection [36]. The only most plausible explanation would be the decreasing prevalence of tobacco smoking since the 1980s in Hong Kong [20], although there is only a moderately strong association between tobacco smoking and the risk of oesophageal adenocarcinoma [37]. We were not able to assess the incidence trends of oesophageal adenocarcinoma in Shanghai due to lack of complete data on histology. An 
earlier study from a high-risk region of China has shown virtually no cases of oesophageal adenocarcinoma in this area [38]. Previous reports from a high-risk area of Iran also suggested that oesophageal adenocarcinoma remained uncommon in this area $[39,40]$. Nevertheless, considering the fact that the two major risk factors for oesophageal adenocarcinoma, obesity and reflux, are becoming more common in Asian populations, together with the lessons from Western countries, a rise in the incidence of oesophageal adenocarcinoma is expected in the coming decades in Asia. However, the link between the changing prevalence of reflux, obesity and $H$. pylori infection and the increasing incidence of oesophageal adenocarcinoma has been mainly generated in Western populations. The aetiology of oesophageal adenocarcinoma in Asian countries, which may differ from that in Western societies, remains to be better understood in the future.

The present study has some strengths and limitations. The data from the cancer registers used were of good quality in terms of completeness of coverage and accuracy, which lend validity to the findings. In addition, the time trends in the incidence of oesophageal cancer were further assessed by histological type in several populations. We also performed age-periodcohort analyses to estimate the overall annual percentage changes (net drifts) in ASRs, which represent the temporal trend with adjustment for age and non-linear birth cohort effects. Therefore, the net drifts may be considered superior to the annual percentage changes in cross-sectional ASRs when interpreting the time trends of disease rates, although the net drifts are virtually analogous to the latter $[17,18]$. A limitation of this study is that the analyses were restricted only to selected cancer registers containing data for the 20 -year study period. Furthermore, we could perform analyses by histological type in only some countries or regions (Hong Kong, Japan, Singapore and Israel) due to lack of complete information on histological typing elsewhere. Thus, the results may not be representative for all Asian countries, or even for all involved countries, e.g. data from Shanghai not representing the 
whole China. Racial-ethnic disparities, e.g. Jews versus non-Jews in Israel or Chinese versus non-Chinese in Singapore, were not within the scope of this study, but need to be addressed in future studies. Finally, any trends in women are difficult to interpret given the low incidence rates, resulting in limited statistical precision. Possible explanations for a lower risk of oesophageal squamous cell carcinoma in women may be lower prevalence of smoker and heavy alcohol use in women, while the reasons for a more striking male predominance of oesophageal adenocarcinoma remain incompletely understood $[4,41]$.

In conclusion, the incidence of oesophageal cancer has decreased in some, but not all Asian populations from 1988 to 2007 . The decrease is attributable to a decline in the incidence of oesophageal squamous cell carcinoma, which in turn is likely to be due to the decreasing prevalence in tobacco smoking. Oesophageal adenocarcinoma remains uncommon in Asian countries. However, there appears to be a trend towards a rise in the incidence of oesophageal adenocarcinoma in some Asian populations. This increase might be associated with the increasing prevalence of reflux and obesity and the decreasing prevalence of $H$. pylori infection, in these populations. More attention needs to be paid to a possible increase in the incidence of oesophageal adenocarcinoma in Asia in the future. 


\section{Competing interests statement}

None declared.

\section{Acknowledgements}

This study was supported by the Swedish Research Council and the Swedish Cancer Society. The funding bodies had no role in the study design, the collection, analysis, and interpretation of data, or the writing of the article and the decision to submit it for publication. 


\section{References}

[1] J. Ferlay, I. Soerjomataram, M. Ervik, R. Dikshit, S. Eser, C. Mathers, M. Rebelo, D.M. Parkin, D. Forman, F. Bray, GLOBOCAN 2012 v1.0, Cancer Incidence and Mortality Worldwide: IARC CancerBase No. 11, International Agency for Research on Cancer, Lyon, France, 2013.

[2] M. Arnold, I. Soerjomataram, J. Ferlay, D. Forman, Global incidence of oesophageal cancer by histological subtype in 2012, Gut 64(3) (2015) 381-7.

[3] A.K. Rustgi, H.B. El-Serag, Esophageal carcinoma, N Engl J Med 371(26) (2014) 2499-509.

[4] J. Lagergren, P. Lagergren, Recent developments in esophageal adenocarcinoma, CA Cancer J Clin 63(4) (2013) 232-48.

[5] G. Edgren, H.O. Adami, E. Weiderpass, O. Nyren, A global assessment of the oesophageal adenocarcinoma epidemic, Gut 62(10) (2013) 1406-14.

[6] C.C. Abnet, D.A. Corley, N.D. Freedman, F. Kamangar, Diet and upper gastrointestinal malignancies, Gastroenterology 148(6) (2015) 1234-1243 e4.

[7] R.Y. Chung, C.M. Schooling, B.J. Cowling, G.M. Leung, How does socioeconomic development affect risk of mortality? An age-period-cohort analysis from a recently transitioned population in China, Am J Epidemiol 171(3) (2010) 345-56.

[8] M.K. Mallath, D.G. Taylor, R.A. Badwe, G.K. Rath, V. Shanta, C.S. Pramesh, R. Digumarti, P. Sebastian, B.B. Borthakur, A. Kalwar, S. Kapoor, S. Kumar, J.L. Gill, M.A. Kuriakose, H. Malhotra, S.C. Sharma, S. Shukla, L. Viswanath, R.T. Chacko, J.L. Pautu, K.S. Reddy, K.S. Sharma, A.D. Purushotham, R. Sullivan, The growing burden of cancer in India: epidemiology and social context, Lancet Oncol 15(6) (2014) e205-12.

[9] D. Forman, F. Bray, D.H. Brewster, C. Gombe Mbalawa, B. Kohler, M. Piñeros, E. SteliarovaFoucher, R. Swaminathan, J. Ferlay, Cancer Incidence in Five Continents, Vol. X, IARC Scientific Publication No. 164, International Agency for Research on Cancer, Lyon, 2014.

[10] M.P. Curado, B. Edwards, H.R. Shin, H. Storm, J. Ferlay, M. Heanue, P. Boyle, Cancer Incidence in Five Continents, Vol. IX, IARC Scientific Publications No. 160, International Agency for Research on Cancer, Lyon, 2007.

[11] D.M. Parkin, S.L. Whelan, J. Ferlay, L. Teppo, D.B. Thomas, Cancer Incidence in Five Continents, Vol. VIII, IARC Scientific Publications No. 155, International Agency for Research on Cancer, Lyon, 2002.

[12] D.M. Parkin, S.L. Whelan, J. Ferlay, L. Raymond, J. Young, Cancer Incidence in Five Continents, Vol. VII, IARC Scientific Publications No. 143, International Agency for Research on Cancer, Lyon, 1997. [13] United Nations, Department of Economic and Social Affairs, Population Division, World Population Prospects: The 2010 Revision, United Nations, New York, 2011.

[14] O.B. Ahmad, C. Boschi-Pinto, A.D. Lopez, C.J.L. Murray, R. Lozano, M. Inoue, Age Standardization of Rates: A New WHO Standard. GPE Discussion Paper Series: No.31, World Health Organization, Geneva, 2001.

[15] M.P. Fay, E.J. Feuer, Confidence intervals for directly standardized rates: a method based on the gamma distribution, Stat Med 16(7) (1997) 791-801.

[16] SAS Institute Inc, SAS/STAT 12.1 User's Guide, SAS Institute Inc., Cary, NC.

[17] P.S. Rosenberg, D.P. Check, W.F. Anderson, A web tool for age-period-cohort analysis of cancer incidence and mortality rates, Cancer Epidemiol Biomarkers Prev 23(11) (2014) 2296-302.

[18] P.S. Rosenberg, W.F. Anderson, Age-period-cohort models in cancer surveillance research: ready for prime time?, Cancer Epidemiol Biomarkers Prev 20(7) (2011) 1263-8.

[19] Institute for Health Metrics and Evaluation (IHME), Tobacco Burden Visualization. $<$ http://vizhub.healthdata.org/tobacco>, 2014).

[20] H.K.D.o.H. Hong Kong Tobacco Control Office, Pattern of smoking in Hong Kong. <http://www.tco.gov.hk/english/infostation/infostation sta 01.html>, 2015 (accessed 15

October.2015). 
[21] S. Tsugane, Alcohol, smoking, and obesity epidemiology in Japan, J Gastroenterol Hepatol 27 Suppl 2 (2012) 121-6.

[22] World Health Organisation, Global status report on alcohol and health 2014, World Health Organisation, Geneva, 2014, p. 267.

[23] I. Oze, K. Matsuo, K. Wakai, C. Nagata, T. Mizoue, K. Tanaka, I. Tsuji, S. Sasazuki, M. Inoue, S. Tsugane, D. Research Group for the, J. Evaluation of Cancer Prevention Strategies in, Alcohol drinking and esophageal cancer risk: an evaluation based on a systematic review of epidemiologic evidence among the Japanese population, Jpn J Clin Oncol 41(5) (2011) 677-92.

[24] M.L. Fernandes, A. Seow, Y.H. Chan, K.Y. Ho, Opposing trends in incidence of esophageal squamous cell carcinoma and adenocarcinoma in a multi-ethnic Asian country, Am J Gastroenterol 101(7) (2006) 1430-6.

[25] Y.K. Yee, T.K. Cheung, A.O. Chan, M.F. Yuen, B.C. Wong, Decreasing trend of esophageal adenocarcinoma in Hong Kong, Cancer Epidemiol Biomarkers Prev 16(12) (2007) 2637-40.

[26] A. Shibata, T. Matsuda, W. Ajiki, T. Sobue, Trend in incidence of adenocarcinoma of the esophagus in Japan, 1993-2001, Jpn J Clin Oncol 38(7) (2008) 464-8.

[27] C.L. Lu, H.C. Lang, J.C. Luo, C.C. Liu, H.C. Lin, F.Y. Chang, S.D. Lee, Increasing trend of the incidence of esophageal squamous cell carcinoma, but not adenocarcinoma, in Taiwan, Cancer Causes Control 21(2) (2010) 269-74.

[28] S. Shiota, S. Singh, A. Anshasi, H.B. El-Serag, Prevalence of Barrett's Esophagus in Asian Countries: A Systematic Review and Meta-analysis, Clin Gastroenterol Hepatol 13(11) (2015) 1907-18.

[29] Institute for Health Metrics and Evaluation (IHME), Overweight and Obesity Viz. $<$ http://vizhub.healthdata.org/obesity >, 2014).

[30] H.B. El-Serag, S. Sweet, C.C. Winchester, J. Dent, Update on the epidemiology of gastrooesophageal reflux disease: a systematic review, Gut 63(6) (2014) 871-80.

[31] K.Y. Ho, Y.H. Chan, J.Y. Kang, Increasing trend of reflux esophagitis and decreasing trend of Helicobacter pylori infection in patients from a multiethnic Asian country, Am J Gastroenterol 100(9) (2005) 1923-8.

[32] S.L. Lim, W.T. Goh, J.M. Lee, T.P. Ng, K.Y. Ho, G.I.S.G. Community Medicine, Changing prevalence of gastroesophageal reflux with changing time: longitudinal study in an Asian population, J

Gastroenterol Hepatol 20(7) (2005) 995-1001.

[33] A. Newnham, M.J. Quinn, P. Babb, J.Y. Kang, A. Majeed, Trends in oesophageal and gastric cancer incidence, mortality and survival in England and Wales 1971-1998/1999, Aliment Pharmacol Ther 17(5) (2003) 655-64.

[34] M. Lindblad, W. Ye, A. Lindgren, J. Lagergren, Disparities in the classification of esophageal and cardia adenocarcinomas and their influence on reported incidence rates, Ann Surg 243(4) (2006) 47985.

[35] V.P. Tan, B.C. Wong, W.M. Wong, W.K. Leung, D. Tong, M.F. Yuen, R. Fass, Gastroesophageal Reflux Disease: Cross-Sectional Study Demonstrating Rising Prevalence in a Chinese Population, J Clin Gastroenterol (2015).

[36] B. Xia, H.H. Xia, C.W. Ma, K.W. Wong, F.M. Fung, C.K. Hui, C.K. Chan, A.O. Chan, K.C. Lai, M.F. Yuen, B.C. Wong, Trends in the prevalence of peptic ulcer disease and Helicobacter pylori infection in family physician-referred uninvestigated dyspeptic patients in Hong Kong, Aliment Pharmacol Ther 22(3) (2005) 243-9.

[37] M.B. Cook, F. Kamangar, D.C. Whiteman, N.D. Freedman, M.D. Gammon, L. Bernstein, L.M. Brown, H.A. Risch, W. Ye, L. Sharp, N. Pandeya, P.M. Webb, A.H. Wu, M.H. Ward, C. Giffen, A.G. Casson, C.C. Abnet, L.J. Murray, D.A. Corley, O. Nyren, T.L. Vaughan, W.H. Chow, Cigarette smoking and adenocarcinomas of the esophagus and esophagogastric junction: a pooled analysis from the international BEACON consortium, J Natl Cancer Inst 102(17) (2010) 1344-53.

[38] G.D. Tran, X.D. Sun, C.C. Abnet, J.H. Fan, S.M. Dawsey, Z.W. Dong, S.D. Mark, Y.L. Qiao, P.R. Taylor, Prospective study of risk factors for esophageal and gastric cancers in the Linxian general population trial cohort in China, Int J Cancer 113(3) (2005) 456-63. 
[39] F. Islami, F. Kamangar, K. Aghcheli, S. Fahimi, S. Semnani, N. Taghavi, H.A. Marjani, S. Merat, S. Nasseri-Moghaddam, A. Pourshams, M. Nouraie, M. Khatibian, B. Abedi, M.H. Brazandeh, R. Ghaziani, M. Sotoudeh, S.M. Dawsey, C.C. Abnet, P.R. Taylor, R. Malekzadeh, Epidemiologic features of upper gastrointestinal tract cancers in Northeastern Iran, Br J Cancer 90(7) (2004) 1402-6.

[40] S. Semnani, A. Sadjadi, S. Fahimi, M. Nouraie, M. Naeimi, J. Kabir, H. Fakheri, H. Saadatnia, M.R. Ghavamnasiri, R. Malekzadeh, Declining incidence of esophageal cancer in the Turkmen Plain, eastern part of the Caspian Littoral of Iran: a retrospective cancer surveillance, Cancer Detect Prev 30(1) (2006) 14-9.

[41] S.H. Xie, J. Lagergren, The Male Predominance in Esophageal Adenocarcinoma, Clin Gastroenterol Hepatol 14(3) (2016) 338-347 e1. 


\section{Table 1}

Crude and age-standardised incidence rates (ASRs) with 95\% confidence intervals (CIs) of oesophageal cancer per 100000 person-years in selected Asian countries and calendar periods during 1988-2007

\begin{tabular}{|c|c|c|c|c|c|c|}
\hline \multirow{2}{*}{ Calendar periods } & \multicolumn{3}{|c|}{ Males } & \multicolumn{3}{|c|}{ Females } \\
\hline & $\mathrm{N}$ & Crude rate $(95 \% \mathrm{CI})$ & $\operatorname{ASR}(95 \% \mathrm{CI})$ & $\mathrm{N}$ & Crude rate $(95 \% \mathrm{CI})$ & $\operatorname{ASR}(95 \% \mathrm{CI})$ \\
\hline \multicolumn{7}{|l|}{ China (Shanghai) } \\
\hline $1988-1992$ & 2855 & $15.71(15.14,16.30)$ & $14.29(13.75,14.86)$ & 1338 & $7.70(7.29,8.12)$ & $5.53(5.23,5.84)$ \\
\hline 1993-1997 & 2355 & $14.34(13.77,14.94)$ & $11.20(10.74,11.68)$ & 1189 & $7.51(7.09,7.95)$ & $4.63(4.37,4.91)$ \\
\hline $1998-2002$ & 2291 & $14.40(13.81,15.00)$ & $10.96(10.50,11.44)$ & 965 & $6.22(5.84,6.63)$ & $3.63(3.40,3.88)$ \\
\hline 2003-2007 & 2017 & $12.96(12.40,13.54)$ & $7.79(7.45,8.15)$ & 791 & $5.16(4.81,5.53)$ & $2.42(2.25,2.60)$ \\
\hline \multicolumn{7}{|c|}{ China (Hong Kong SAR) } \\
\hline 1988-1992 & 2179 & $14.91(14.29,15.55)$ & $15.72(15.05,16.42)$ & 565 & $4.05(3.72,4.40)$ & $3.62(3.33,3.94)$ \\
\hline 1993-1997 & 2145 & $13.78(13.21,14.38)$ & $13.03(12.48,13.60)$ & 553 & $3.58(3.29,3.89)$ & $2.95(2.71,3.22)$ \\
\hline $1998-2002$ & 2030 & $12.41(11.88,12.97)$ & $10.54(10.08,11.01)$ & 455 & $2.69(2.45,2.95)$ & $2.02(1.83,2.22)$ \\
\hline $2003-2007$ & 1780 & $10.89(10.39,11.41)$ & $7.96(7.59,8.34)$ & 493 & $2.78(2.54,3.03)$ & $1.84(1.67,2.02)$ \\
\hline \multicolumn{7}{|c|}{ Japan (Miyagi, Nagasaki, and Osaka) } \\
\hline 1988-1992 & 4114 & $13.46(13.05,13.87)$ & $11.34(11.00,11.7)$ & 955 & $3.00(2.81,3.19)$ & $1.98(1.85,2.11)$ \\
\hline 1993-1997 & 5256 & $17.07(16.61,17.53)$ & $12.26(11.93,12.60)$ & 1100 & $3.41(3.21,3.62)$ & $1.95(1.84,2.07)$ \\
\hline $1998-2002$ & 6530 & $21.15(20.64,21.67)$ & $12.96(12.65,13.28)$ & 1273 & $3.91(3.70,4.13)$ & $1.96(1.85,2.08)$ \\
\hline
\end{tabular}


2003-2007

7595

$24.45(23.9,25.01)$

2053

$5.59(5.35,5.83)$

$5.12(4.90,5.35)$

1993-1997

1998-2002

2003-2007

Thailand (Chiang Mai)

1988-1992

1993-1997

1998-2002

2003-2007

Singapore

1988-1992

1993-1997

1998-2002

2003-2007

Philippines (Manila)

1988-1992

143

158

1993-1997
$1.34(1.13,1.57)$

$1.27(1.08,1.49)$
$12.93(12.63,13.23)$

$10.39(9.91,10.89)$

$9.41(8.98,9.86)$

$8.18(7.82,8.54)$

$6.59(6.30,6.89)$

$2.54(1.96,3.22)$

$2.36(1.85,2.98)$

$2.49(1.98,3.09)$

$1.60(1.23,2.05)$

$8.67(7.83,9.56)$

$6.63(5.98,7.33)$

$5.79(5.22,6.41)$

$4.10(3.66,4.57)$

$3.49(2.92,4.12)$
$3.49(2.90,4.16)$

1494

$4.51(4.28,4.74)$

$2.08(1.97,2.20)$
1389

1406

1384

1272
$4.47(4.24,4.71)$

$4.16(3.94,4.38)$

$3.74(3.55,3.94)$

$3.18(3.01,3.36)$

$1.41(1.03,1.87)$

$1.07(0.76,1.47)$

$0.82(0.56,1.17)$

$0.57(0.36,0.87)$

$2.23(1.89,2.62)$

$1.54(1.28,1.85)$

$1.37(1.12,1.65)$

$0.95(0.76,1.18)$

$0.80(0.65,0.98)$

$0.65(0.52,0.81)$
$7.82(7.40,8.26)$

$7.16(6.78,7.56)$

$5.36(5.07,5.65)$

$4.25(4.02,4.50)$

$1.74(1.27,2.31)$

$1.20(0.85,1.65)$

$0.85(0.58,1.22)$

$0.48(0.30,0.74)$

$2.65(2.24,3.11)$

$1.66(1.37,2.00)$

$1.40(1.15,1.68)$

$0.82(0.65,1.02)$

$1.96(1.56,2.42)$

$1.59(1.26,1.96)$ 


\begin{tabular}{|c|c|c|c|c|c|c|}
\hline 1998-2002 & 195 & $1.51(1.30,1.73)$ & $3.47(2.96,4.03)$ & 61 & $0.46(0.35,0.59)$ & $0.90(0.68,1.16)$ \\
\hline 2003-2007 & 193 & $1.34(1.16,1.55)$ & $2.97(2.52,3.46)$ & 83 & $0.56(0.44,0.69)$ & $1.05(0.82,1.30)$ \\
\hline \multicolumn{7}{|l|}{ Israel } \\
\hline 1988-1992 & 220 & $1.87(1.63,2.13)$ & $1.91(1.66,2.18)$ & 161 & $1.35(1.15,1.58)$ & $1.18(1.00,1.38)$ \\
\hline 1993-1997 & 352 & $2.56(2.30,2.85)$ & $2.71(2.43,3.02)$ & 212 & $1.51(1.32,1.73)$ & $1.23(1.07,1.41)$ \\
\hline $1998-2002$ & 358 & $2.36(2.12,2.62)$ & $2.38(2.14,2.65)$ & 228 & $1.47(1.29,1.68)$ & $1.08(0.94,1.24)$ \\
\hline 2003-2007 & 357 & $2.17(1.95,2.41)$ & $2.09(1.87,2.32)$ & 243 & $1.45(1.27,1.64)$ & $1.05(0.92,1.20)$ \\
\hline
\end{tabular}

* Standardised to the World Health Organization (WHO) World Standard Population 2000. 


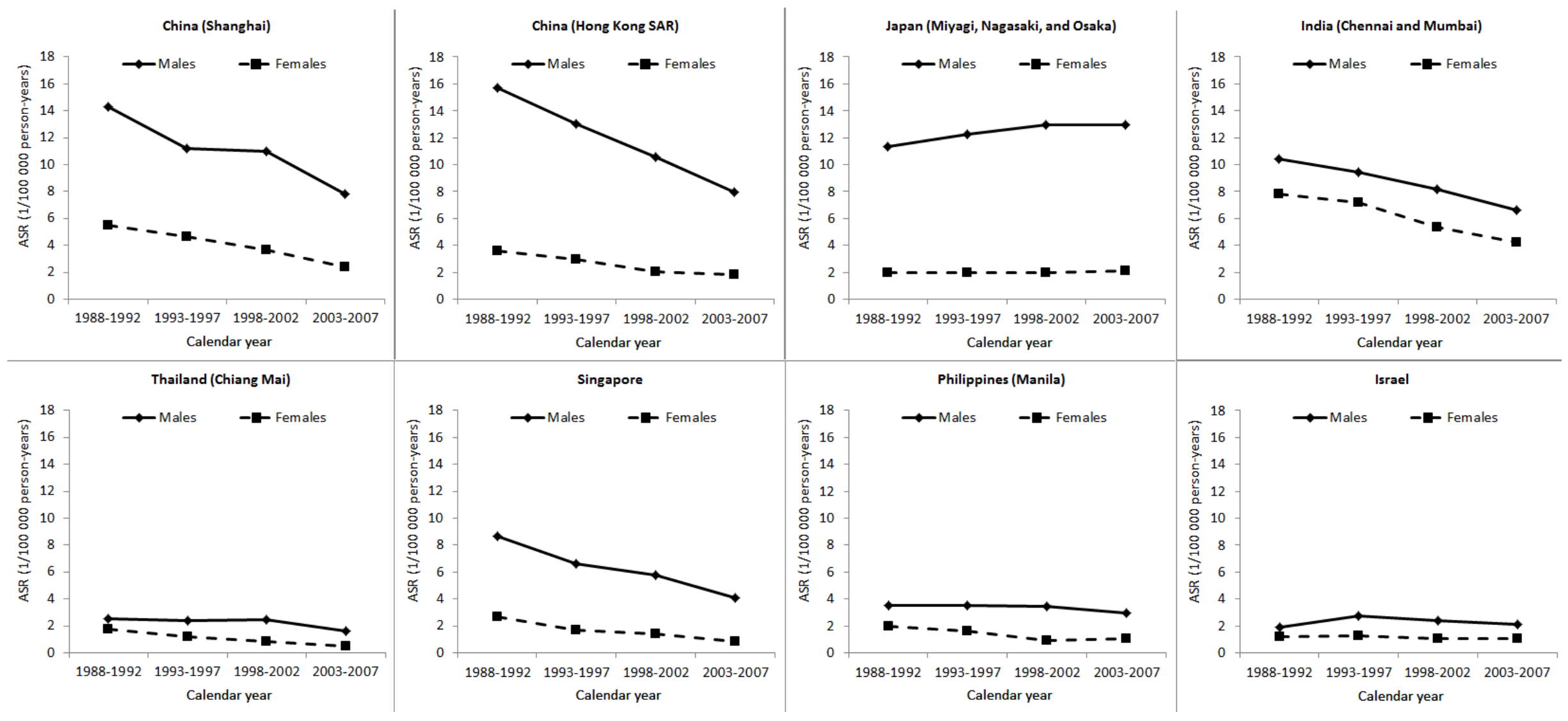

Fig. 1. Age-standardised incidence rates of oesophageal cancer by sex in selected Asian populations in 1988-2007 using the WHO World Standard Population 2000 as reference. 


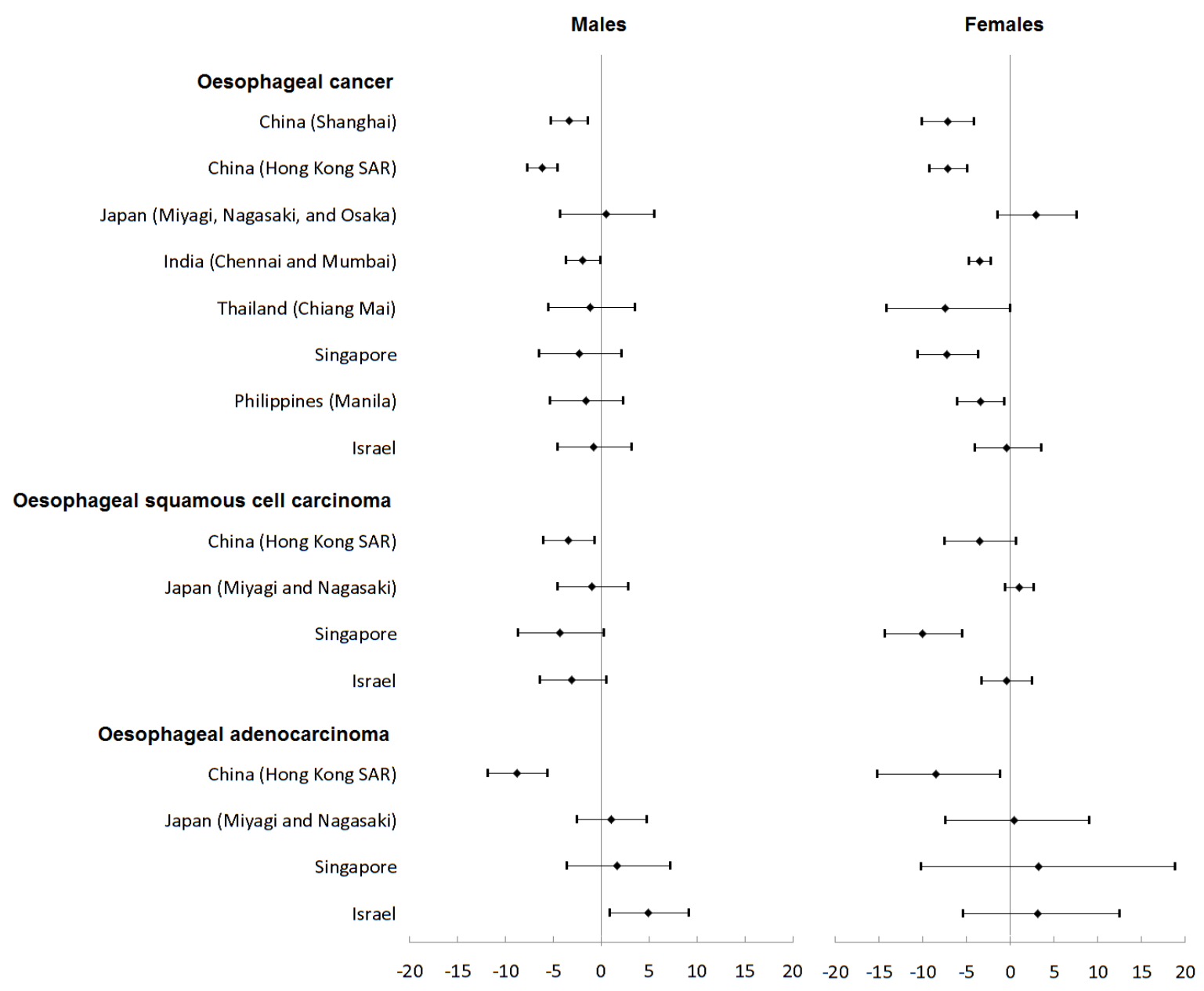

Fig. 2. The overall annual percentage changes (net drifts) and their $95 \%$ confidence intervals in the incidence of oesophageal cancer by sex and histological type in selected Asian populations in 1988-2007. 


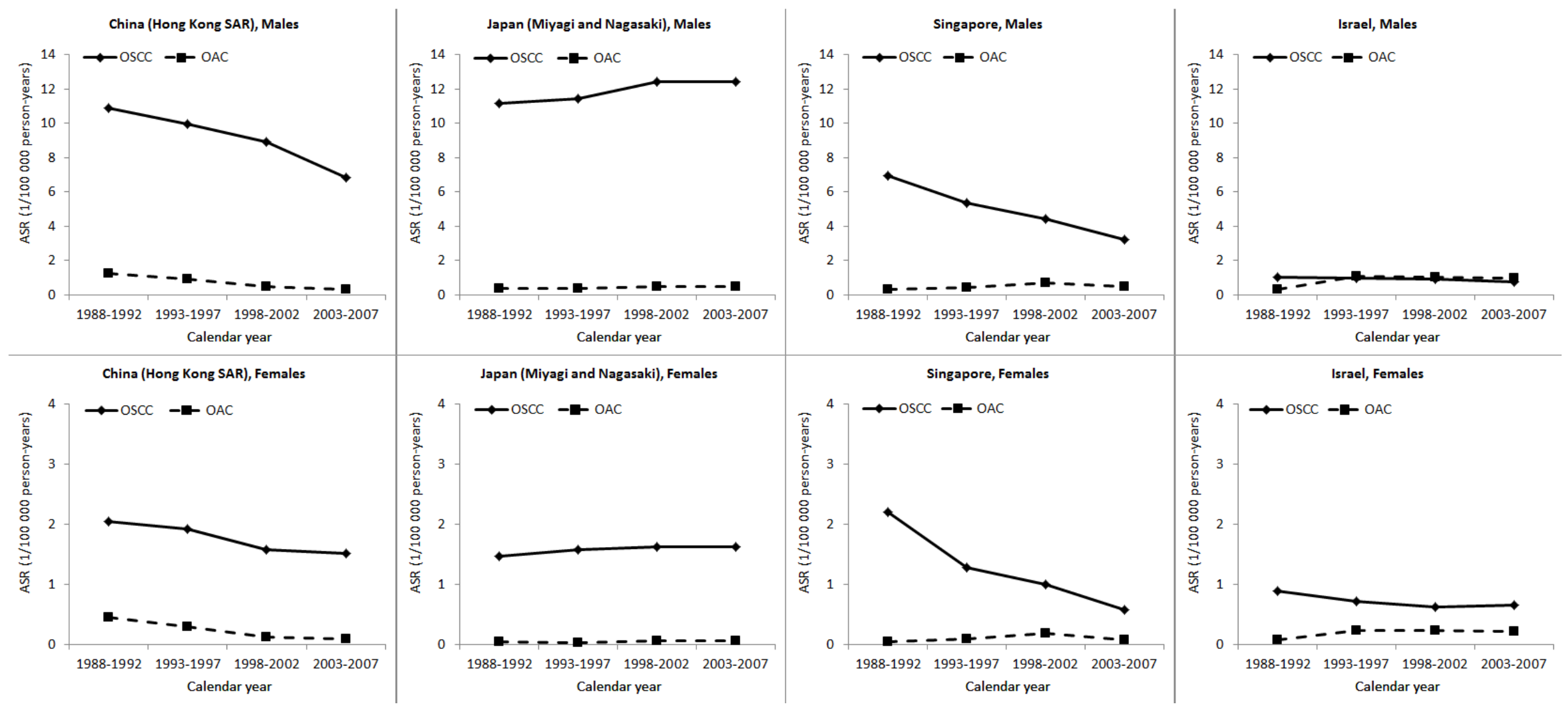

Fig. 3. Age-standardised incidence rates (ASRs) of oesophageal squamous cell carcinoma (OSCC) and adenocarcinoma (OAC) by sex in selected Asian populations in 1988-2007 using the WHO World Standard Population 2000 as reference. 
Supplementary Table 1. Crude and age-standardised incidence rates (ASRs) with 95\% confidence intervals (CIs) of oesophageal squamous cell carcinoma per 100000 person-years by country and calendar period in Asia, 1988-2007

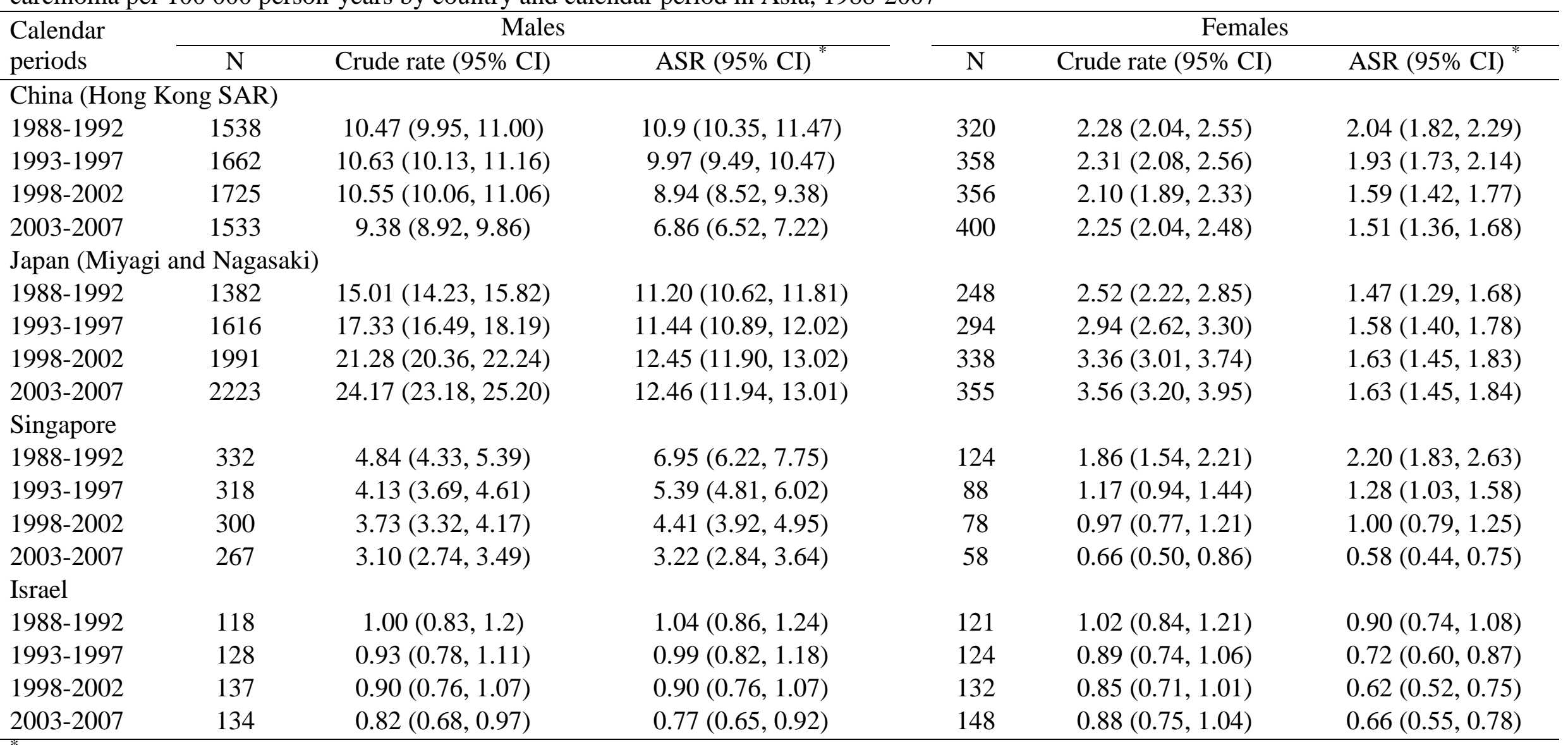

Standardised to the World Health Organization (WHO) World Standard Population 2000. 
Supplementary Table 2. Crude and age-standardised incidence rates (ASRs) with 95\% confidence intervals (CIs) of oesophageal adenocarcinoma per 100000 person-years by country and calendar period in Asia, 1988-2007

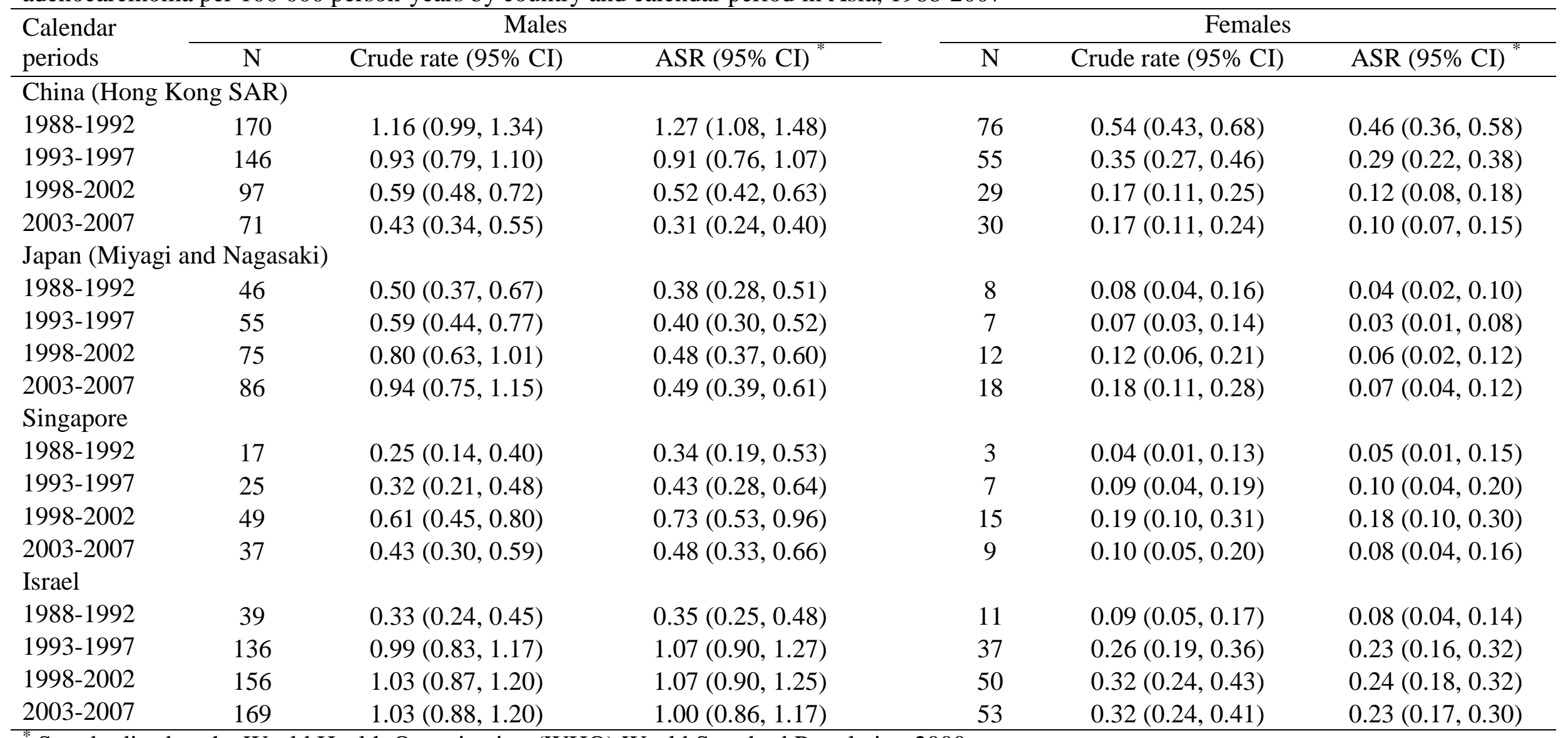

Standardised to the World Health Organization (WHO) World Standard Population 2000. 


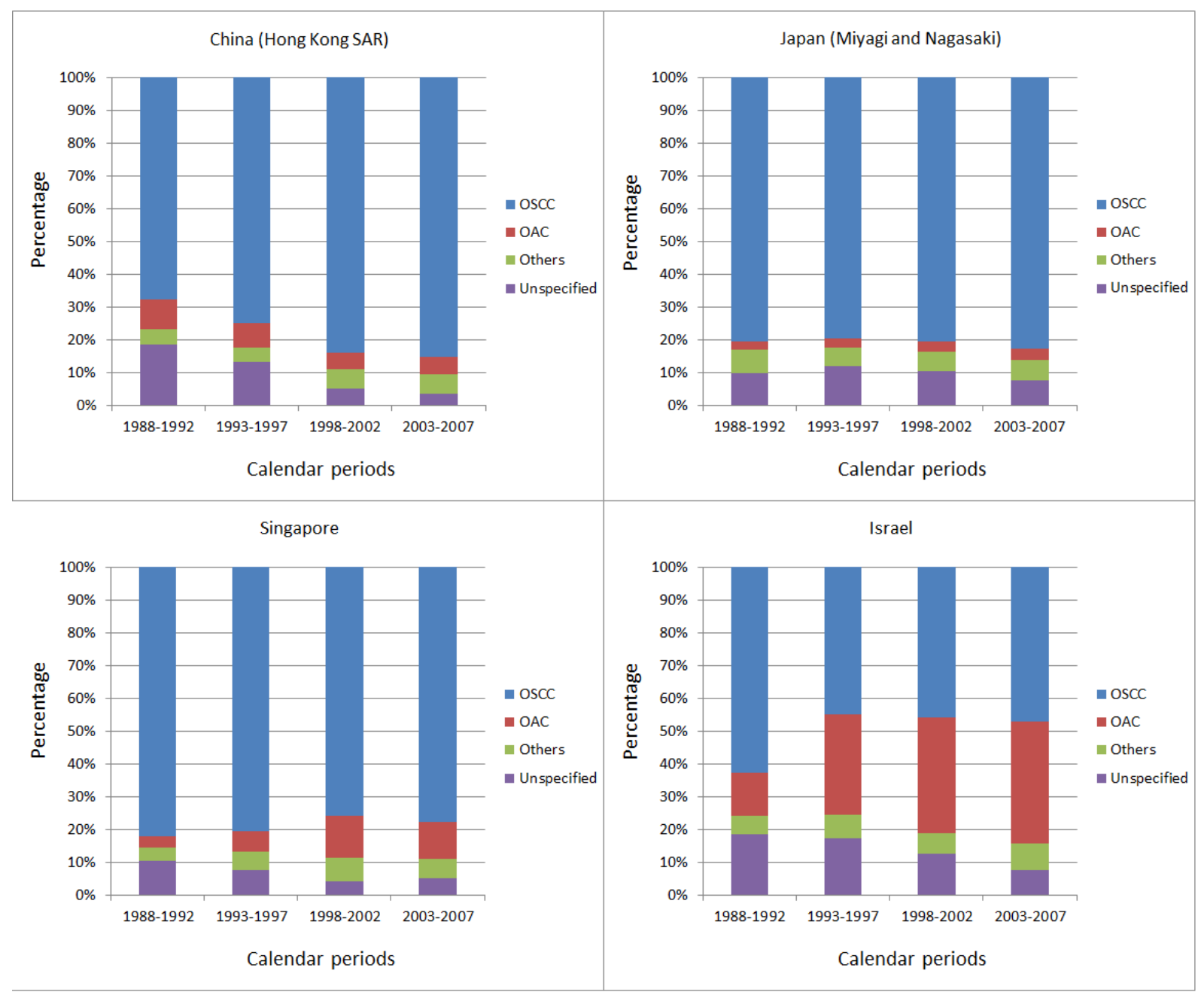

Supplementary Figure 1. Percentages of histological types of oesophageal cancer in selected Asian populations by calendar period during 1988-2007. 


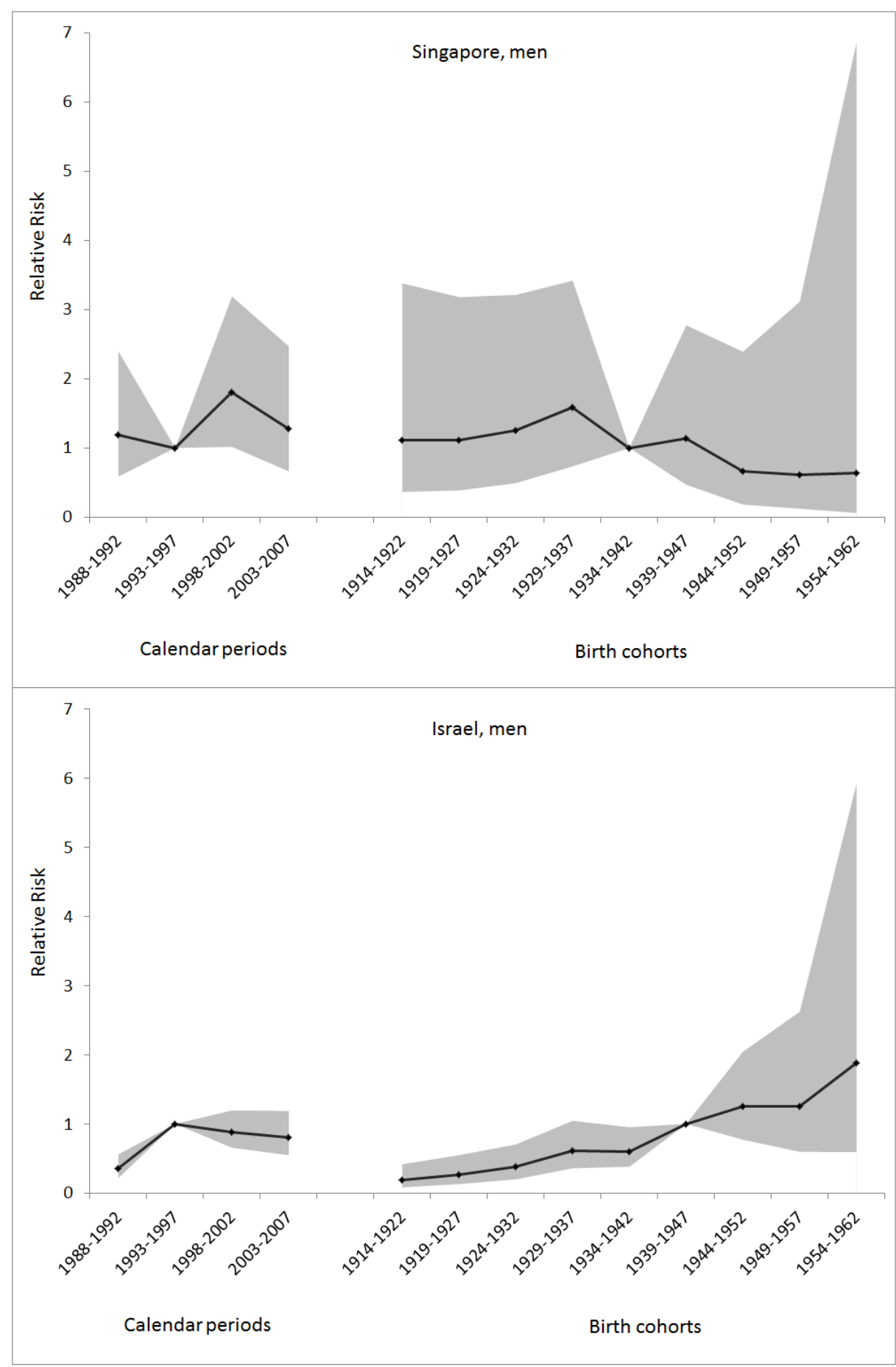

Supplementary Figure 2. Period and cohort effects and their $95 \%$ confidence intervals obtained from age-period-cohort analyses for the incidence rates of oesophageal adenocarcinoma in men in Singapore and Israel. 